\title{
Impact of Moving From a Widespread to Multisite Pain Definition on Other Fibromyalgia Symptoms
}

\author{
LINDA E. DEAN, ${ }^{1}$ LESLEY ARNOLD, ${ }^{2}$ LESLIE CROFFORD, ${ }^{3}$ ROBERT BENNETT, ${ }^{4}$ DON GOLDENBERG, ${ }^{5}$ \\ MARY-ANN FITZCHARLES, ${ }^{6}$ EDUARDO S. PAIVA, ${ }^{7}$ ROLAND STAUD, ${ }^{8}$ DAN CLAUW,${ }^{9}$ \\ PIERCARLO SARZI-PUTTINI, ${ }^{10}$ GARETH T. JONES, ${ }^{1}$ ABIMBOLA AYORINDE, ${ }^{1}$ \\ ELISA FLÜß, ${ }^{1}$ MARCUS BEASLEY, ${ }^{1}$ AND GARY J. MACFARLANE ${ }^{1}$
}

Objective. To investigate whether associations between pain and the additional symptoms associated with fibromyalgia are different in persons with chronic widespread pain (CWP) compared to multisite pain (MSP), with or without joint areas.

Methods. Six studies were used: 1958 British birth cohort, Epidemiology of Functional Disorders, Kid Low Back Pain, Managing Unexplained Symptoms (Chronic Widespread Pain) in Primary Care: Involving Traditional and Accessible New Approaches, Study of Health and its Management, and Women's Health Study (WHEST; females). MSP was defined as the presence of pain in $\geq 8$ body sites in adults ( $\geq 10$ sites in children) indicated on 4 -view body manikins, conducted first to include joints (positive joints) and second without (negative joints). The relationship between pain and fatigue, sleep disturbance, somatic symptoms, and mood impairment was assessed using logistic regression. Results are presented as odds ratios (ORs) with 95\% confidence intervals (95\% CIs).

Results. There were 34,818 participants across the study populations (adults age range $42-56$ years, male $43-51 \%$ [excluding WHEST], and CWP prevalence 12-17\%). Among those reporting MSP, the proportion reporting CWP ranged between $62 \%$ and $76 \%$. Among those reporting the symptoms associated with fibromyalgia, there was an increased likelihood of reporting pain, the magnitude of which was similar regardless of the definition used. For example, within WHEST, reporting moderate/severe fatigue (Chalder fatigue scale 4-11) was associated with a $>5$-fold increase in likelihood of reporting pain (CWP OR 5.2 [95\% CI 3.9-6.9], MSP-positive joints OR 6.5 [95\% CI 5.0-8.6], and MSPnegative joints OR 6.5 [95\% CI 4.7-9.0]).

Conclusion. This large-scale study demonstrates that regardless of the pain definition used, the magnitude of association between pain and other associated symptoms of fibromyalgia is similar. This finding supports the continued collection of both when classifying fibromyalgia, but highlights the fact that pain may not require to follow the definition outlined within the 1990 American College of Rheumatology criteria.

\section{INTRODUCTION}

The understanding and classification of pain syndromes, such as fibromyalgia, have been evolving. Since its early beginnings as fibrositis, interest in fibromyalgia has steadily increased, especially with regard to strategies used for the classification of affected individuals, which remain controversial.

Supported by the Analgesic, Anesthetic, and Addiction Clinical Trial Translations, Innovations, Opportunities, and Networks initiative.

${ }^{1}$ Linda E. Dean, PhD, Gareth T. Jones, PhD, Abimbola Ayorinde, PhD, Elisa Flüß, PhD, Marcus Beasley, MSc, Gary J. Macfarlane, MBChB, PhD, CStat, MD, FFPHM: University of Aberdeen, Aberdeen, UK; ${ }^{2}$ Lesley Arnold, MD: University of Cincinnati, Cincinnati, Ohio; ${ }^{3}$ Leslie Crofford, MD: Vanderbilt University, Nashville, Tennessee; ${ }^{4}$ Robert Bennett, MD, FRCP: Oregon Health and Science University, Portland; ${ }^{5}$ Don Goldenberg, MD: Newton-Wellesley Hospital, Newton, Massachusetts; ${ }^{6}$ Mary-Ann Fitzcharles, MBChB, MD: McGill
The initial American College of Rheumatology (ACR)endorsed 1990 classification criteria for fibromyalgia required the presence of contralateral (left side, right side, above waist, and below waist) and axial body pain, in addition to pain on palpation in at least 11 (of 18) specified anatomical points (1). Although these criteria resulted in many clinical and epidemiologic studies, which improved the understanding of the etiology and

University, Montreal, Quebec, Canada; ${ }^{7}$ Eduardo S. Paiva, MD, FACR: Universidade Federal do Paraná, Curitiba, Brazil; ${ }^{8}$ Roland Staud, MD: University of Florida, Gainesville; ${ }^{\mathbf{9}}$ Dan Clauw, MD: University of Michigan, Ann Arbor; ${ }^{10}$ Piercarlo Sarzi-Puttini, MD: University of Milan, Milan, Italy. Address correspondence to Gary J. Macfarlane, $\mathrm{MBChB}$, $\mathrm{PhD}$, CStat, MD, FFPHM, Epidemiology Group, Institute of Applied Health Sciences, University of Aberdeen, School of Medicine, Medical Sciences, and Nutrition, Health Sciences Building, Foresterhill, Aberdeen, AB25 2ZD, UK. E-mail: g.j.macfarlane@abdn.ac.uk.

Submitted for publication August 17, 2016; accepted in revised form January 31, 2017. 


\section{Significance \& Innovations}

- We have developed a description of multisite pain that gives a similar prevalence to chronic widespread pain, defined as the presence of $\geq 8$ pain sites.

- The magnitude of association between pain and the symptoms associated with fibromyalgia is similar, regardless of whether joint areas were included in the definition of multisite pain or not.

- The continued collection of information on both pain and associated symptoms when classifying fibromyalgia remains important, but the definition of pain may not necessarily require following the definition outlined in the 1990 American College of Rheumatology criteria for fibromyalgia.

outcome of fibromyalgia, there were issues with operationalization in the clinical setting, partly due to the inconsistency in examination for tender points (2). These pain-centered criteria also failed to consider other common, nonpain, fibromyalgia symptoms such as fatigue. Generally, the agreement between the ACR 1990 classification criteria and clinical fibromyalgia diagnosis is approximately $75 \%$ (3).

In response to these limitations, the proposed 2010 preliminary diagnostic criteria (and the 2011 modification for research) moved fibromyalgia from a pain-focused to a symptom-focused condition $(4,5)$. In doing so, the pain aspect of the criteria moved from requiring pain to be widespread to being multisite, and the previously required tender-point examination was removed. In addition to the presence of chronic multisite pain (MSP; lasting $\geq 3$ months), the proposed diagnostic criteria also required the presence of additional symptoms associated with fibromyalgia: fatigue, waking unrefreshed, and somatic and cognitive symptoms. The 2010 diagnostic criteria have provoked considerable debate and have been criticized as being difficult to operationalize (6), and alternative criteria have also been proposed (7). In addition, it is also important to note that all 3 proposed criteria for fibromyalgia (1990, preliminary 2010, and 2011 modification) were developed in populations of rheumatic disease patients.

In this context, the current analysis was undertaken as part of the Analgesic, Anesthetic, and Addiction Clinical Trial Translations, Innovations, Opportunities, and Networks (ACTTION) initiative to develop a new taxonomy for pain conditions. Specifically informing the development of a taxonomy for fibromyalgia, the aims of the current analysis were to assess and compare the descriptive epidemiology of pain reporting in the general population, using widespread pain and MSP definitions (with and without the inclusion of joints), to assess the relationship between these pain definitions and the other associated symptoms of fibromyalgia, and due to the predominance of fibromyalgia in females, to determine whether there is any sexual dimorphism in the reporting of these symptoms.

\section{PATIENTS AND METHODS}

Study populations. Six existing studies (5 with general populations and 1 with a school-based sampling frame) were used for the current post hoc analysis. In all studies participants were asked if they had experienced, during the previous month, aches or pains lasting for at least 1 day. Positive responders were consequently invited to mark the specific areas in which this pain had occurred on 4-view blank body manikins (which were thereafter coded with a maximum of 35 marked sites) (Figure 1). Participants were also asked whether the pain had lasted at least 3 months (indicating chronic pain). Manikin coding was conducted within each study by trained research staff. This procedure has previously shown high interrater reliability in the classification of widespread pain (8).

The Epidemiology of Functional Disorders (EPIFUND) is a prospective cohort study consisting of a random sample of subjects ages 25-65 years from 3 areas of the UK (9). Data from the initial recruitment survey, collected by means of self-complete postal questionnaire, were used for the current analysis. Information was collected on sleep problems (estimation of sleep problem scale [10]), mood symptoms (hospital anxiety and depression scales [11]), and the presence of somatic symptoms (somatic symptom scale [12]).

The Kid Low Back Pain (Kid LBP) study is a prospective cohort study conducted across secondary schools in Cheshire and North Derbyshire, UK (13). School children ages 11-14 years were eligible for the study. Data from the initial self-complete questionnaire were used for the current analysis.

The Managing Unexplained Symptoms (Chronic Widespread Pain) in Primary Care: Involving Traditional and Accessible New Approaches (MUSICIAN) study is a $2 \times 2$ factorial randomized controlled trial, undertaken to investigate the management of chronic widespread pain (CWP) (14). General practitioner practices across Aberdeen City and North Cheshire County of the UK were used as a sampling frame, from which randomly selected individuals ages $\leq 25$ years were sent a self-complete screening questionnaire by mail. Those data were used in the current analysis.

The Study of Health and its Management (SHAMA) is a cross-sectional study of individuals residing in the Grampian region of the UK, ages $\leq 25$ years (15) who selfcompleted a questionnaire sent by mail. The questionnaire included a measurement of quality of life (Short Form 36 health survey [SF-36]) (16).

The National Child Development (1958 British birth cohort) study included all children born in Great Britain during a single week in March 1958. Subjects have been followed over the course of their lifetime (17). In 2003 (when the cohort was 45 years old), participants selfcompleted a questionnaire that included a measure of psychological distress (general health questionnaire) (18). 

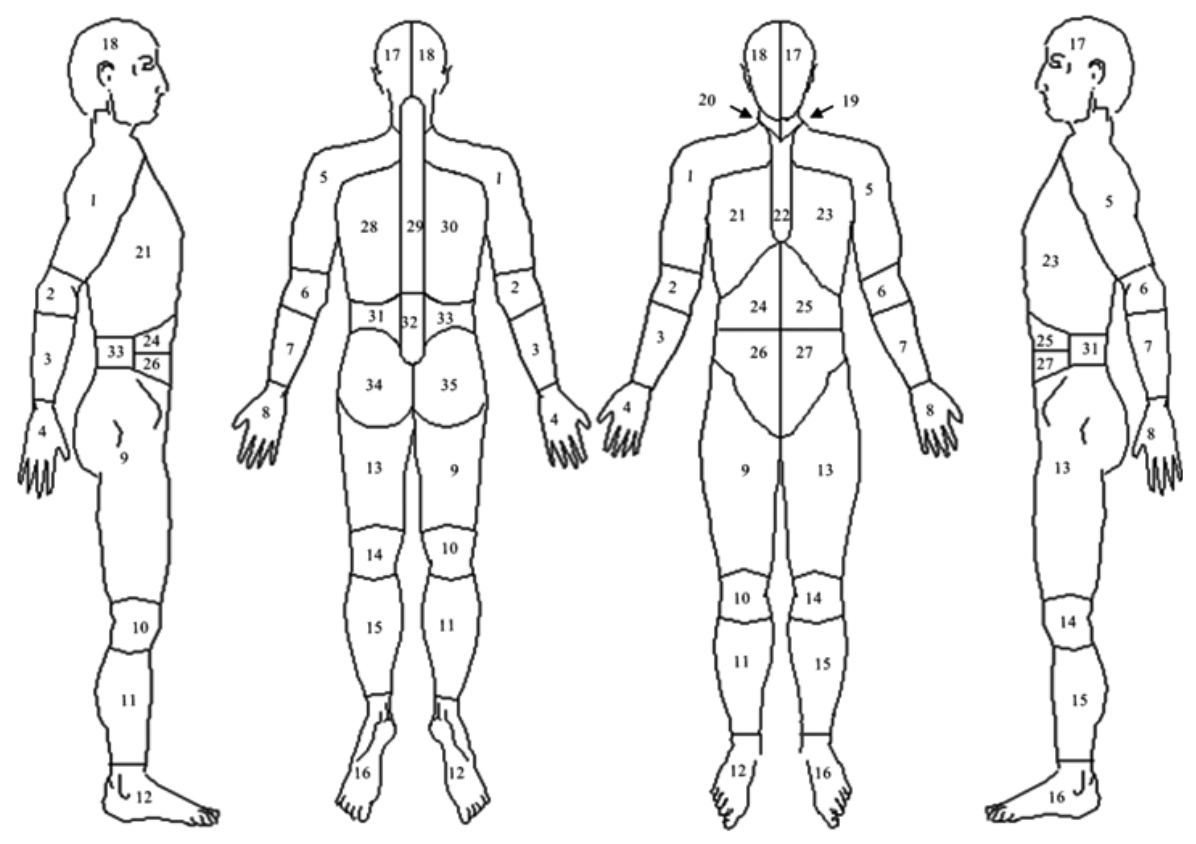

Figure 1. Four-view body manikins with scoring.

The Women's Health Study (WHEST) is a cross-sectional study of females, ages $\leq 25$ years, resident in the Grampian region of the UK, who were sent a self-complete questionnaire by post. This questionnaire included measures of fatigue (Chalder fatigue scale [19]), sleep disturbance (estimation of sleep problem scale [10]), somatic symptom reporting (somatic symptom scale [12]), and depression (patient health questionnaire-9 [20]).

Consent was obtained from potential participants within each original study as per their individual governance arrangements. All data used within the current study were fully anonymized.

Classification of pain. Participants were classified as having widespread pain (contralateral pain and pain in the axial skeleton), CWP (widespread pain lasting $\geq 3$ months), regional pain (pain that was not widespread), or no pain (1). The cumulative distribution of the number of painful body sites was examined within each of the 6 study populations and was used to determine definitions of MSP that produced the closest prevalence to CWP (according to the ACR 1990 definition). As an example, if $\mathrm{X} \%$ of the study population reported CWP (ACR 1990), we chose the number of sites whose cumulative prevalence was closest to $100-\mathrm{X} \%$ as the definition of MSP. This process was conducted twice, once to include all body locations, hereafter defined as MSP-positive joints, and secondly with the exclusion of all 10 joint areas, hereafter defined as MSP-negative joints.

Statistical analysis. The characteristics of each of the populations, and the subgroups fulfilling the CWP, MSPpositive joints, and MSP-negative joints definitions, were examined using simple descriptive statistics, reported as proportions for categorical variables and population mean \pm SD for continuous factors. The relationship between the additional symptoms associated with fibromyalgia, and the reporting of each of these pain definitions, were assessed using multiple binary logistic regression models. Fatigue was assessed using the Chalder fatigue scale, categorized by standard cutoffs into none (score 0), mild (scores 1-3) and moderate/severe (scores 4-11) (19), or the SF-36 vitality score. Due to the skewed distribution of the data, a pragmatic approach was chosen to categorize the SF-36 vitality into tertiles, high (scores 81-100), mid (scores 58-80), and poor (scores 0-57) (16). All analyses were thereafter conducted comparing the poorest tertile to the middle and highest combined, to maximize patient number while also retaining a clinically meaningful group. Sleep problems were assessed using the estimation of sleep problem scale, categorized by standard cutoffs as no sleep problems (scores 0-11) or sleep problems (scores 12-20) (10), and the presence of any somatic symptoms was assessed by the somatic symptom scale (12). Lastly, emotional/mental health was assessed by 4 different instruments. The general health questionnaire was subdivided into tertiles of high (score 0), mid (scores 1-2), and poor (scores 3-12) (18). The patient health questionnaire was subdivided into mild, moderate, moderately severe, and severe according to standard instrument cutoffs (21). The SF-36 mental health score was divided into tertiles due to skewed distribution (poor [score 0-79], mid [scores 80-86], and high [scores 87-100]) (16), and analysis was conducted on the poorest versus the mid and high groups. Lastly the hospital anxiety and depression scales were grouped into scores of no, borderline, and clinical anxiety/depression according to standard instrument cutoffs (11).

Additionally, to assess potential sexual dimorphism, of those reporting MSP-positive joints, differences in the reporting of the additional symptoms associated with fibromyalgia were assessed, stratified by sex. For all 


\begin{tabular}{|c|c|c|c|c|c|c|}
\hline & SHAMA & WHEST & EPIFUND & MUSICIAN & Kid LBP & 1958 BBC \\
\hline No. & 1,579 & 2,303 & 6,244 & 14,680 & 1,440 & 8,572 \\
\hline Male, \% & 45 & 0 & 43 & 43 & 46 & 51 \\
\hline $\begin{array}{l}\text { Age, mean } \pm \text { SD years } \\
\text { Type of pain, no. (\%) }\end{array}$ & $55 \pm 15$ & $53 \pm 15$ & $46 \pm 11$ & $56 \pm 16$ & $12 \pm 0.9$ & $42(-)$ \\
\hline None & $782(50)$ & $1,028(45)$ & $2,343(38)$ & $5,424(37)$ & $390(27)$ & $4,019(47)$ \\
\hline Regional & $570(36)$ & 884 (38) & $2,709(43)$ & $6,601(45)$ & $863(60)$ & $3,318(39)$ \\
\hline Widespread & $30(2)$ & $54(2)$ & $129(2)$ & $239(2)$ & $109(8)$ & $179(2)$ \\
\hline Chronic widespread & 197 (12) & $337(15)$ & $1,063(17)$ & $2,416(16)$ & $78(5)$ & $1,056(12)$ \\
\hline
\end{tabular}

logistic regression models, results are presented as odds ratios (ORs) and 95\% confidence intervals (95\% CIs). All statistical analysis was undertaken using STATA software, version 13.

\section{RESULTS}

Characteristics of study populations. The total number of participants was 34,818 , and the number from each study varied from 1,440 (Kid LBP) to 14,680 (MUSICIAN). Excluding WHEST, which contained only females, the proportion of male participants ranged between $43 \%$ and $51 \%$ (Table 1). The mean age range within the adult cohorts was narrow, 42-56 years, while children in the Kid LBP study had a mean age of 12 years. The prevalence of CWP within the adult studies varied between $12 \%$ and $17 \%$ and was lower within the only child population $(7 \%)$ (Table 1).

MSP definition. Although the prevalence of CWP differed between the adult study populations, the number of corresponding sites defining both MSP-positive joints and MSP-negative joints definitions was consistent across all studies, namely $\geq 8$ sites (Table 2 ). The corresponding MSP (positive and negative joints) definitions were different only within the child population, which used at least 10 sites.

There was substantial overlap between CWP and MSP groups (Table 2). Within the adult populations, of persons satisfying the definition of MSP-positive joints, between $62 \%$ and $72 \%$ also fulfilled the CWP definition. Among those meeting the MSP-negative joints definition, between $68 \%$ and $79 \%$ also met the definition of CWP. Among the child population, those also fulfilling the CWP definition were substantially lower (MSP positive $39 \%$ and MSP negative $42 \%$ ). There was consistency in the mean age and sex proportions across the adult population groups (Table 3). For example, within the EPIFUND study, the mean \pm SD age in both CWP and MSP-positive joints groups was $48 \pm 11$ years and in the MSP-negative joints groups was $47 \pm 11$ years. The proportion of males in the same study was $36 \%, 35 \%$, and $30 \%$, for CWP, MSP-positive joints, and MSP-negative joints groups, respectively.

Relationship between pain and the additional symptoms associated with fibromyalgia. The populations meeting the CWP, MSP-positive joints, and MSP-negative joints criteria were characterized by their reporting of fatigue, sleep problems, somatic symptoms and mental/emotional health.

Fatigue. Fatigue was assessed within 2 studies (SHAMA and WHEST). Those who reported fatigue were more likely to report pain, regardless of the pain definition used (Table 4). The magnitude of this association was greater with MSP definitions. For example, within the WHEST study, compared to no fatigue, reporting moderate/severe fatigue was associated with a 5-fold increase in the odds of CWP (OR 5.2 [95\% CI 3.9-6.9]) and a 6.5-fold increase in the odds of MSP (MSP-positive joints OR 6.5 [95\% CI 5.0-8.6]; MSP-negative joints OR 6.5 [95\% CI 4.7-9.0]) (Table 4).

\begin{tabular}{|c|c|c|c|c|c|c|c|c|c|c|c|c|}
\hline \multirow[b]{2}{*}{ MSP definition } & \multicolumn{2}{|c|}{ SHAMA } & \multicolumn{2}{|c|}{ WHEST } & \multicolumn{2}{|c|}{ EPIFUND } & \multicolumn{2}{|c|}{ MUSICIAN } & \multicolumn{2}{|c|}{ Kid LBP } & \multicolumn{2}{|c|}{$1958 \mathrm{BBC}$} \\
\hline & Pos. & Neg. & Pos. & Neg. & Pos. & Neg. & Pos. & Neg. & Pos. & Neg. & Pos. & Neg. \\
\hline No. of sites for definition & $\geq 8$ & $\geq 8$ & $\geq 8$ & $\geq 8$ & $\geq 8$ & $\geq 8$ & $\geq 8$ & $\geq 8$ & $\geq 10$ & $\geq 10$ & $\geq 8$ & $\geq 8$ \\
\hline No. of those fulfilling MSP & 219 & 136 & 397 & 235 & 1,061 & 568 & 2,715 & 1,623 & 84 & 26 & 1,110 & 633 \\
\hline Percent who also had CWP & 62 & 68 & 66 & 75 & 72 & 75 & 68 & 79 & 39 & 42 & 66 & 74 \\
\hline \multicolumn{13}{|c|}{$\begin{array}{l}\text { * Chronic widespread pain (CWP) defined according to the American College of Rheumatology } 1990 \text { criteria. SHAMA = Study of Health } \\
\text { and its Management; WHEST = Women's Health Study; EPIFUND = Epidemiology of Functional Disorders; MUSICIAN = Managing Unex- } \\
\text { plained Symptoms (Chronic Widespread Pain) in Primary Care: Involving Traditional and Accessible New Approaches; Kid LBP = Kid } \\
\text { Low Back Pain; BBC = British birth cohort; MSP = multisite pain; Pos. = positive; Neg. = negative. }\end{array}$} \\
\hline
\end{tabular}


Sleep problems. Sleep problems were assessed within 2 studies (WHEST and EPIFUND). Those who reported sleep problems had a 4-fold increased odds of reporting pain (Table 4), and this result was consistent across pain definitions. For example, within the EPIFUND study, CWP was OR 4.1 (95\% CI 3.6-4.8), MSP-positive joints OR 4.1 (95\% CI 3.6-4.8), and MSP-negative joints OR 4.2 (95\% CI 3.5-5.0) (Table 4).

Somatic symptoms. Somatic symptoms were assessed within 2 studies (WHEST and EPIFUND). The reporting of any somatic symptom was associated with an increased odds of reporting pain. The magnitude of this association was similar across all pain definitions for each individual symptom. For example, within the EPIFUND study, reporting breathing difficulties was associated with approximately a 2.5-fold increase in the odds of reporting pain: CWP OR 2.5 (95\% CI 2.1-2.8), MSP-positive joints OR 2.6 (95\% CI 2.33.0), and MSP-negative joints OR 2.7 (95\% CI 2.3-3.3) (Table 4). The only notable difference in pain reporting was demonstrated within the somatic symptom of digit pain, in which the odds of pain reporting were larger for MSPpositive joints compared to the other pain groups: CWP OR 4.2 (95\% CI 3.7-4.9), MSP-positive joints OR 5.2 (95\% CI 4.5-5.9), and MSP-negative joints OR 4.6 (95\% CI 3.9-5.5) (Table 4).

Mental and emotional health. Mental/emotional health was assessed within 4 studies (1958 British birth cohort, WHEST, SHAMA, and EPIFUND). Generally, reporting impaired mental health was associated with an increased odds of reporting pain (Table 4). The magnitude of this association increased as the severity of mental health problems worsened but was consistent across pain definitions. As an example, within the EPIFUND study and using the HADS scale, reporting borderline depression was associated with at least a 2.5-fold increase in the odds of pain (CWP OR 2.5 [95\% CI 2.1-3.1], MSP-positive joints OR 2.5 [95\% CI 2.0-2.99], and MSP-negative joints OR 2.9 [95\% CI 2.3-3.7]), while reporting clinical depression was associated with a $<4$-fold increase in the odds of pain (CWP OR 4.5 [95\% CI 3.6-5.5], MSP-positive joints OR 4.2 [95\% CI 3.4-5.2], and MSP-negative joints OR 4.7 [95\% CI 3.7-6.0]) (Table 4).

Relationship between pain and the additional symptoms associated with fibromyalgia, stratified by sex. With the aim of assessing potential sexual dimorphism, stratification was used to assess sex differences in the association between pain reporting (MSP-positive joints) and other additional symptoms associated with fibromyalgia. Reporting fatigue was associated with an increased odds of reporting pain regardless of sex (Table 5). Among females, compared to those reporting moderate/high vitality (SF-36 vitality score 58-100), those reporting poor vitality (score $0-57$ ) had a 5-fold increase in the odds of also reporting MSP-positive joints (OR 5.1 [95\% CI 3.3-7.7]). Within males, the odds of pain reporting in the presence of poor vitality was OR 4.4 (95\% CI 2.7-7.1). Those who reported sleep problems demonstrated an increased odds of reporting MSP-positive joints, and the magnitude of this association was similar across both males and females: male OR 4.4 (95\% CI 3.5-5.6); female OR 3.8 (95\% CI 


\begin{tabular}{|c|c|c|c|c|c|c|}
\hline \multirow[b]{2}{*}{ Measure (study population) } & \multicolumn{2}{|r|}{ CWP } & \multicolumn{2}{|c|}{ MSP-positive joints } & \multicolumn{2}{|c|}{ MSP-negative joints } \\
\hline & No. & OR (95\% CI) & No. & OR (95\% CI) & No. & OR (95\% CI) \\
\hline \multicolumn{7}{|l|}{ Fatigue } \\
\hline \multicolumn{7}{|c|}{$\begin{array}{l}\text { SF-36 vitality vs. medium/high tertile: score } \\
\text { 58-100 (SHAMA) }\end{array}$} \\
\hline Poor: score $0-57$ & 1,579 & $5.3(3.8-7.5)+$ & 1,579 & $4.9(3.5-6.7) \dagger$ & 1,579 & $5.1(3.4-7.6)+$ \\
\hline \multicolumn{7}{|l|}{ Chalder fatigue scale vs. absent (WHEST) } \\
\hline Mild: score $1-3$ & 2,178 & $2.9(2.1-3.9) \dagger$ & 2,178 & $3.0(2.2-4.0)+$ & 2,178 & $3.0(2.1-4.3) \dagger$ \\
\hline Moderate/severe: score 4-11 & 2,178 & $5.2(3.9-6.9)+$ & 2,178 & $6.5(5.0-8.6) \dagger$ & 2,178 & $6.5(4.7-9.0)+$ \\
\hline \multicolumn{7}{|l|}{ Sleep problems } \\
\hline \multicolumn{7}{|c|}{ Estimation of sleep problems vs. no (WHEST) } \\
\hline Yes & 2,258 & $3.6(2.9-4.7)+$ & 2,258 & $3.8(3.0-4.7)+$ & 2,258 & $4.3(3.3-5.7) \dagger$ \\
\hline \multicolumn{7}{|c|}{ Estimation of sleep problems vs. no (EPIFUND) } \\
\hline Yes & 6,039 & $4.1(3.6-4.8)+$ & 6,027 & $4.1(3.6-4.8) \dagger$ & 6,039 & $4.2(3.5-5.0)$ \\
\hline \multicolumn{7}{|l|}{ Somatic symptoms present } \\
\hline \multicolumn{7}{|c|}{ Somatic symptom scale, yes vs. no (WHEST) } \\
\hline Breathing difficulties & 2,291 & $2.7(2.1-3.4) \dagger$ & 2,291 & $3.1(2.5-3.9)+$ & 1,195 & $2.0(1.5-2.6) \dagger$ \\
\hline Menstrual cramps & 2,276 & $2.4(1.9-3.1)+$ & 2,276 & $2.3(1.8-2.8) \dagger$ & 1,187 & $2.1(1.6-2.8)+$ \\
\hline Lost voice & 2,290 & $1.3(1.0-1.8)$ & 2,290 & $1.6(1.2-2.1) \dagger$ & 1,195 & $1.1(0.8-1.7)$ \\
\hline Difficulty swallowing & 2,298 & $2.9(2.2-3.7)+$ & 2,298 & $3.0(2.3-3.8)+$ & 1,200 & $1.9(1.4-2.6) \dagger$ \\
\hline Memory loss & 2,293 & $2.9(2.2-4.0)+$ & 2,293 & $3.4(2.5-4.5) \dagger$ & 1,194 & $2.6(1.8-3.6) \dagger$ \\
\hline Frequently vomit & 2,294 & $2.4(1.6-3.6)+$ & 2,294 & $2.5(1.7-3.7)+$ & 1,197 & $1.9(1.2-3.0) \neq$ \\
\hline Digit pain & 2,289 & $4.8(3.7-6.2)+$ & 2,289 & $5.7(4.4-7.2)+$ & 1,192 & $2.3(1.7-3.1)+$ \\
\hline \multicolumn{7}{|c|}{ Somatic symptom scale, yes vs. no (EPIFUND) } \\
\hline Breathing difficulties & 6,196 & $2.5(2.1-2.8) \dagger$ & 6,210 & $2.6(2.3-3.0)+$ & 6,210 & $2.7(2.3-3.3) \dagger$ \\
\hline Menstrual cramps & 3,426 & $1.7(1.4-2.0)+$ & 3,432 & $1.7(1.4-2.0) \dagger$ & 3,432 & $1.8(1.5-2.3) \dagger$ \\
\hline Lost voice & 6,214 & $1.5(1.3-1.8)+$ & 6,228 & $1.4(1.2-1.7)+$ & 6,228 & $1.5(1.2-1.8) \neq$ \\
\hline Difficulty swallowing & 5,326 & $2.7(2.3-3.2)+$ & 5,338 & $2.7(2.3-3.2) \dagger$ & 5,338 & $2.6(2.1-3.2) \dagger$ \\
\hline Memory loss & 6,208 & $2.5(2.1-3.0) \dagger$ & 6,221 & $2.4(2.0-2.9)+$ & 6,221 & $2.4(1.9-3.0) \dagger$ \\
\hline Frequently vomit & 6,185 & $2.8(2.2-3.7) \dagger$ & 6,198 & $2.8(2.1-3.7)+$ & 6,198 & $3.4(2.5-4.7) \dagger$ \\
\hline Digit pain & 6,187 & $4.2(3.7-4.9) \dagger$ & 6,201 & $5.2(4.5-5.9)+$ & 6,201 & $4.6(3.9-5.5) \dagger$ \\
\hline \multicolumn{7}{|l|}{ Emotional/mental health } \\
\hline \multicolumn{7}{|c|}{$\begin{array}{l}\text { General health questionnaire vs. top tertile: } \\
\text { score } 0 \text { (1958 British birth cohort) }\end{array}$} \\
\hline Mid: score 1-2 & 8,283 & $1.5(1.3-1.8) \dagger$ & 8,283 & $1.3(1.1-1.5) \ddagger$ & 8,283 & $1.3(1.1-1.7) \dagger$ \\
\hline Poor: score 3-12 & 8,283 & $2.3(1.9-2.7) \dagger$ & 8,283 & $2.2(1.9-2.6) \dagger$ & 8,283 & $2.5(2.1-3.1) \dagger$ \\
\hline \multicolumn{7}{|c|}{$\begin{array}{l}\text { Patient health questionnaire-9 vs. no depression } \\
\text { (WHEST) }\end{array}$} \\
\hline Mild depression & 2,215 & $3.4(2.6-4.5)+$ & 2,215 & $3.3(2.5-4.5) \dagger$ & 1,145 & $2.1(1.5-3.1)+$ \\
\hline Moderate depression & 2,215 & $7.1(5.0-10.1) \dagger$ & 2,215 & $5.7(3.9-8.2) \dagger$ & 1,145 & $4.1(2.7-6.4) \dagger$ \\
\hline Moderately severe depression & 2,215 & $10.5(6.4-17.3) \dagger$ & 2,215 & $10.5(6.4-17.4) \dagger$ & 1,145 & $7.9(4.3-14.3)+$ \\
\hline Severe depression & 2,215 & $11.2(6.0-21.1) \dagger$ & 2,215 & $9.1(4.8-17.3) \dagger$ & 1,145 & $7.9(3.6-17.2)+$ \\
\hline \multicolumn{7}{|c|}{$\begin{array}{l}\text { SF-36 mental health vs. medium/high tertile: } \\
\text { score } 80-100 \text { (SHAMA) }\end{array}$} \\
\hline Poor (score 0-79) & 1,578 & $2.9(2.1-3.9) \dagger$ & 1,578 & $3.3(2.4-4.5) \dagger$ & 1,578 & $3.2(2.2-4.7) \dagger$ \\
\hline \multicolumn{7}{|c|}{$\begin{array}{l}\text { Hospital anxiety and depression scale vs. none: } \\
\text { score 0-7 (EPIFUND) }\end{array}$} \\
\hline Anxiety borderline: score 8-10 & 6,161 & $1.9(1.6-2.3) \dagger$ & 6,148 & $1.8(1.5-2.1) \dagger$ & 6,161 & $2.1(1.7-2.7) \dagger$ \\
\hline Anxiety present: score 11-21 & 6,161 & $3.2(2.7-3.7)+$ & 6,148 & $3.2(2.7-3.8)+$ & 6,161 & $3.3(2.7-3.7) \dagger$ \\
\hline Depression borderline: score 8-10 & 6,169 & $2.5(2.1-3.1)+$ & 6,155 & $2.5(2.0-3.0)+$ & 6,169 & $2.9(2.3-3.7) \dagger$ \\
\hline Depression present: score 11-21 & 6,169 & $4.5(3.6-5.5)+$ & 6,155 & $4.2(3.4-5.2)+$ & 6,161 & $4.7(3.7-6.0)+$ \\
\hline $\begin{array}{l}\text { * Values are the number of subjects includ } \\
\text { dence interval; SF-36 }=\text { Short Form } 36 \text { healt } \\
\text { Epidemiology of Functional Disorders. } \\
+P<0.001 . \\
\text { \& } P<0.05 \text {. }\end{array}$ & & & & $T=W_{0}$ & & $\begin{array}{l}\% \text { CI }=95 \% \text { confi- } \\
\text { Study; EPIFUND = }\end{array}$ \\
\hline
\end{tabular}

3.2-4.6) (Table 5). Additionally, those reporting somatic symptoms were more likely to report MSP-positive joints (Table 5), and the magnitude of this result was similar across sex. For example, reporting breathing difficulties was associated with approximately a 2.5-fold increase in the odds of pain in both males (OR 2.4 [95\% CI 1.9-3.1]) and females (OR 2.6 [95\% CI 2.2-3.1]). Lastly, those who reported impaired mental health were more likely to report 
Table 5. Relationship between MSP (positive joints) and symptoms associated with fibromyalgia, stratified by sex*

\begin{tabular}{|c|c|c|c|c|}
\hline \multirow[b]{2}{*}{ Measure (study population) } & \multicolumn{2}{|r|}{ Males } & \multicolumn{2}{|r|}{ Females } \\
\hline & No. & OR (95\% CI) & No. & OR (95\% CI) \\
\hline \multicolumn{5}{|l|}{ Fatigue } \\
\hline \multicolumn{5}{|c|}{ SF-36 vitality vs. medium/high tertile: score 58-100 (SHAMA) } \\
\hline Poor: score $0-57$ & 705 & $4.4(2.7-7.1) \dagger$ & 874 & $5.1(3.3-7.7) \dagger$ \\
\hline \multicolumn{5}{|l|}{ Sleep problems } \\
\hline \multicolumn{5}{|l|}{ Estimation of sleep problems vs. no (EPIFUND) } \\
\hline Yes & 2,631 & $4.4(3.5-5.6) \dagger$ & 3,408 & $3.8(3.2-4.6) \dagger$ \\
\hline \multicolumn{5}{|l|}{ Somatic symptoms } \\
\hline \multicolumn{5}{|l|}{ Somatic symptom scale, yes vs. no (EPIFUND) } \\
\hline Breathing difficulties & 2,706 & $2.4(1.9-3.1) \dagger$ & 3,504 & $2.6(2.2-3.1) \dagger$ \\
\hline Menstrual cramps & & - & 3,400 & $1.7(1.4-2.0) \dagger$ \\
\hline Lost voice & 2,709 & $1.2(0.8-1.7)$ & 3,519 & $1.3(1.1-1.6) \neq$ \\
\hline Difficulty swallowing & 2,360 & $2.9(2.2-3.9) \dagger$ & 2,975 & $2.5(2.0-3.1) \dagger$ \\
\hline Memory loss & 2,704 & $2.7(2.0-3.6) \dagger$ & 3,517 & $2.3(1.8-2.9) \dagger$ \\
\hline Frequently vomit & 2,686 & $2.8(1.7-4.6) \dagger$ & 3,512 & $2.7(1.9-3.7) \dagger$ \\
\hline Digit pain & 2,699 & $4.8(3.9-6.1) \dagger$ & 3,502 & $5.2(4.4-6.3) \dagger$ \\
\hline \multicolumn{5}{|l|}{ Emotional/mental health } \\
\hline \multicolumn{5}{|c|}{ General health questionnaire vs. top tertile: score 0 (1958 British birth cohort) } \\
\hline Mid: score $1-2$ & 4,046 & $1.1(0.9-1.4)$ & 4,237 & $1.5(1.2-1.9) \dagger$ \\
\hline Poor: score 3-12 & 4,046 & $1.9(1.5-2.4) \dagger$ & 4,237 & $2.5(2.0-3.1) \dagger$ \\
\hline \multicolumn{5}{|c|}{ SF-36 mental health vs. medium/high tertile: score 80-100 (SHAMA) } \\
\hline Poor: score $0-79$ & 705 & $3.3(2.0-5.3) \dagger$ & 873 & $3.2(2.2-4.7) \dagger$ \\
\hline \multicolumn{5}{|c|}{ Hospital anxiety and depression scale vs. none: score 0-7 (EPIFUND) } \\
\hline Anxiety borderline: score $8-10$ & 2,685 & $2.3(1.7-3.0) \dagger$ & 3,476 & $1.6(1.3-2.0) \dagger$ \\
\hline Anxiety present: score $11-21$ & 2,685 & $3.8(2.9-5.0)+$ & 3,476 & $2.7(2.2-3.3) \dagger$ \\
\hline Depression borderline: score $8-10$ & 2,681 & $2.9(2.1-4.1) \dagger$ & 3,488 & $2.3(1.8-2.9) \dagger$ \\
\hline Depression present: score $11-21$ & 2,681 & $5.0(3.6-7.0) \dagger$ & 3,488 & $4.2(3.2-5.5) \dagger$ \\
\hline $\begin{array}{l}\text { * Numbers are the model number. MSP }=\text { multisite pain; OR } \\
\text { SHAMA }=\text { Study of Health and its Management; EPIFUND }= \\
+P<0.001 . \\
\neq P<0.05 .\end{array}$ & $\begin{array}{l}\text { dence } \\
\text { ders. }\end{array}$ & rval; SF-36 = & Fo & 36 health survey; \\
\hline
\end{tabular}

MSP-positive joints regardless of sex (Table 5). For example, reporting borderline depression (compared to no depression) was associated with over a doubling in the odds of pain (male OR 2.9 [95\% CI 2.1-4.1]; female OR 2.3 [95\% CI 1.8-2.9]) and reporting clinical depression was associated with over a quadrupling in the odds of pain (male OR 5.0 [95\% CI 3.6-7.0]; female OR 4.2 [95\% CI 3.2-5.5]).

In addition to the raw comparison of the relationship between pain and the additional associated symptoms of fibromyalgia by sex, potential differences were further assessed using interaction terms. These additional models (data not shown) confirmed that there were no statistically significant interactions between sex and fatigue, sleep problems, somatic symptoms, or mental/emotional health $(P>0.05$ for all).

\section{DISCUSSION}

We have demonstrated that there is little difference, and the magnitude of association was similar between fatigue, sleep disturbance, the reporting of somatic symptoms, or cognitive problems and CWP or MSP (with or without consideration of joint pain). Additionally, there is no indication of systematic differences in the magnitude of these associations by sex.
There are a number of methodologic considerations in interpreting the current findings. First, one of the primary aims of the study was to assess the relationship between alternative pain area definitions (CWP/MSP) and the additional associated symptoms of fibromyalgia. The MSP definition was formulated in such a way as to identify a population that gave a similar prevalence to that of CWP. Due to this coordination, one may expect that both groups would include many of the same individuals, and therefore the study would have only modest power to detect potential differences. The overlap observed was between $60 \%$ and $76 \%$, leaving a substantial number of unique individuals within each group. Additionally, the characteristics of those within the groups differed, pain chronicity being the most apparent. By definition, 100\% of the CWP group experienced chronic pain (pain $\geq 3$ months duration); however, within the MSP groups, this dropped to as low as $77 \%$ (adult population). The similarities shown between groups are therefore not solely due to the overlap of patients, although this overlap will have contributed.

The relationship between pain and the other associated symptoms of fibromyalgia was assessed through bivariate analysis, using studies that contained both a measure of pain (which allowed the classification of CWP and MSP) and at least 1 of the associated symptoms that fibromyalgia 
features. Unfortunately, no single study contained all predictors; therefore, a fully adjusted model could not be created. Although the aim here was to deduce how these features individually predicted pain, future studies assessing whether these relationships remain when controlling for the other symptoms would be valuable.

Additionally, although the magnitude of association between these symptoms and pain was broadly similar across pain definition, there were differences noted across the study and measure used. Although there was little overlap in the instruments across studies, both WHEST and EPIFUND collected identical sleep disturbance and somatic symptom measures. The results from these studies exhibited small differences in the magnitude of association with pain, differences that may largely be due to the study populations themselves. While EPIFUND is a general population survey, WHEST only recruited females, and as females are generally more likely to report symptoms such as insomnia and sleep disturbance (22), these differences are perhaps not surprising. Conversely, multiple instruments were used to measure both fatigue and emotional/mental health. Although differences in the magnitude of association with pain can partly be attributed to the fact that the instruments used, although broadly measuring similar concepts, are not identical and may be expected to be capturing slightly different aspects. Although there were differences in OR across measures, all multiple-level outcomes demonstrated similar doserelationship trends, e.g., the worse the mental/emotional category the greater the likelihood of reporting pain.

Within the current study, 4-view body manikins were used to assess pain areas rather than those areas presented in list form, as they are within the published fibromyalgia criteria $(1,4,5)$. As the 2011 modification, for use within epidemiologic and clinical studies, is a relatively recent development, none of the population studies used in the current analysis included this modification. Pain areas were assessed through the pain manikins across all 6 studies. The number of pain sites needed $(\geq 8)$ to define MSP within the adult studies was identical and is consistent with those proposed within other studies (23).

This large-scale post hoc study, encompassing over 34,000 individuals across multiple studies, is the first to assess the association between different pain area definitions and the relationship with the other symptoms associated with fibromyalgia, and demonstrated similar magnitudes of association, regardless of the definition used. In the absence of gold standard criteria for fibromyalgia, we have shown that there may be multiple ways in which the core feature of this syndrome, namely pain, could be defined, a concept that has been demonstrated in other conditions, such as within the classification of shoulder pain (24). Although CWP is the underpinning concept of the ACR 1990 classification of fibromyalgia, its prevalence differs considerably based on the specific definition used (25). In line with previous studies that sought to compare associations of widespread pain and MSP with known risk factors, the current study supports classification of pain simply by number of sites $(26,27)$.

The original ACR 1990 classification criteria included a definition of pain distribution that is widespread and included joint areas (1), but the more recent 2010/2011 modifications have gone on to classify MSP without considering many of the original joint areas $(4,5)$. Within the current study we aimed to determine whether the reinclusion of joints within an MSP definition would result in differences in the relationship with the additional features of fibromyalgia. The results have shown, in fact, that there is little difference in the relationship between pain and the other features, regardless as to whether joint areas were included or not. Excluding these areas from classification criteria may omit a large proportion of individuals who exhibit pain in multiple sites but due to the specific location of these sites do not meet the current widespread definition $(4,5)$. Future classification criteria for fibromyalgia may consider the reinclusion of joint areas within the definition of pain.

The current study also aimed to determine, among those fulfilling the new definition of MSP, whether there were sex differences in the reporting of the other features of fibromyalgia. Although fibromyalgia is more common in females (particularly when 1990 or clinical diagnoses are used [6]), the current study did not provide evidence of differences in the relationship between pain and these features. This finding is consistent with previous studies which have shown that there is little difference by sex in the reporting of symptoms such as anxiety/depression (28-30) and somatic symptoms (29) among those reporting pain.

Lastly, in addition to including several independent adult populations, the current study also included a study in schoolchildren (Kid LBP study). Within the juvenile population, the prevalence of CWP was lower than within adults, but regional pain was more common. This finding may be explained, in part, by the developmental stage of the study participants and indicates that standard adult definitions of pain may not be suitable for child populations. Furthermore, the classification of widespread pain and MSP may need to be made differently in this group. This conclusion is supported by previous studies that indicated differences in the reporting of fibromyalgia symptoms in young adults (31). Specifically, the additional features of fibromyalgia (notably sleep quality and depressive symptoms) demonstrated stronger association with symptom severity than for widespread pain.

To our knowledge, this is the largest study (using information on over 34,000 individuals across multiple studies) to have assessed the association between pain areas and the other associated symptoms of fibromyalgia in the context of alternative pain definitions. We have demonstrated similar magnitudes of association with chronic widespread pain and MSP (whether or not joint areas are included). Although the results presented here should be further validated within other populations outside the UK, researchers and clinicians alike should be advised that the continued collection of information on the additional associated symptoms of fibromyalgia, in conjunction with pain, remain important when classifying fibromyalgia, but that this pain definition may not necessarily require to follow the distribution outlined by the 1990 ACR criteria for fibromyalgia. 


\section{ACKNOWLEDGMENTS}

The authors thank the investigators and supporters of the 1958 British birth cohort (Medical Research Council), EPIFUND (Arthritis Research UK), Kid LBP (Colt Foundation), MUSICIAN (Arthritis Research UK), SHAMA, and WHEST (British Pain Society-Mildred Clulow Award and National Health Service Grampian), for providing the data necessary for the current analysis.

\section{AUTHOR CONTRIBUTIONS}

All authors were involved in drafting the article or revising it critically for important intellectual content, and all authors approved the final version to be submitted for publication. Dr. Macfarlane had full access to all of the data in the study and takes responsibility for the integrity of the data and the accuracy of the data analysis.

Study conception and design. Arnold, Crofford, Bennett, Macfarlane.

Acquisition of data. Jones, Ayorinde, Flüß, Beasley.

Analysis and interpretation of data. Dean, Goldenberg, Fitzcharles, Paiva, Staud, Clauw, Sarzi-Puttini, Macfarlane.

\section{REFERENCES}

1. Wolfe F, Smythe HA, Yunus MB, Bennett RM, Bombardier C, Goldenberg DL, et al. The American College of Rheumatology 1990 criteria for the classification of fibromyalgia. Arthritis Rheum 1990;33:160-72.

2. Fitzcharles MA, Boulos P. Inaccuracy in the diagnosis of fibromyalgia syndrome: analysis of referrals. Rheumatology (Oxford) 2003;42:263-7.

3. Katz RS, Wolfe F, Michaud K. Fibromyalgia diagnosis: a comparison of clinical, survey, and American College of Rheumatology criteria. Arthritis Rheum 2006;54:169-76.

4. Wolfe F, Clauw DJ, Fitzcharles MA, Goldenberg DL, Katz RS, Mease P, et al. The American College of Rheumatology preliminary diagnostic criteria for fibromyalgia and measurement of symptom severity. Arthritis Care Res (Hoboken) 2010;62:600-10.

5. Wolfe F, Clauw DJ, Fitzcharles MA, Goldenberg DL, Hauser W, Katz RS, et al. Fibromyalgia criteria and severity scales for clinical and epidemiological studies: a modification of the ACR preliminary diagnostic criteria for fibromyalgia. J Rheumatol 2011;38:1113-22.

6. Jones GT, Atzeni F, Beasley M, Flüß E, Sarzi-Puttini P, Macfarlane GJ. The prevalence of fibromyalgia in the general population: a comparison of the American College of Rheumatology 1990, 2010, and modified 2010 classification criteria. Arthritis Rheumatol 2015;67:568-75.

7. Bennett RM, Friend R, Marcus D, Bernstein C, Han BK, Yachoui R, et al. Criteria for the diagnosis of fibromyalgia: validation of the modified 2010 preliminary American College of Rheumatology criteria and the development of alternative criteria. Arthritis Care Res (Hoboken) 2014;66:1364-73.

8. Lacey RJ, Lewis M, Jordan K, Jinks C, Sim J. Interrater reliability of scoring of pain drawings in a self-report health survey. Spine 2005;30:E455-8.

9. McBeth J, Nicholl BI, Cordingley L, Davies KA, Macfarlane GJ. Chronic widespread pain predicts physical inactivity: results from the prospective EPIFUND study. Eur J Pain 2010;14:972-9.

10. Jenkins CD, Stanton B, Niemcryk SJ, Rose RM. A scale for the estimation of sleep problems in clinical research. J Clin Epidemiol 1988;41:313-21.
11. Zigmond AS, Snaith RP. The hospital anxiety and depression scale. Acta Psychiatr Scand 1983;67:361-70.

12. Ohtmer E, DeSouza C. A screening test for somatization disorder. Am J Psychiatry 1985;142:1146-9.

13. Watson KD, Papageorgiou AC, Jones GT, Taylor S, Symmons DP, Silman AJ, et al. Low back pain in schoolchildren: occurrence and characteristics. Pain 2002;97:87-92.

14. Macfarlane GJ, Beasley M, Jones EA, Prescott GJ, Docking R, Keeley P, et al. The prevalence and management of low back pain across adulthood: results from a population-based cross-sectional study (the MUSICIAN study). Pain 2012; 153:27-32.

15. Flüß E, Bond CM, Jones GT, Macfarlane GJ. The re-evaluation of the measurement of pain in population-based epidemiological studies: the SHAMA study. Br J Pain 2015;9: 131-41.

16. Brazier JE, Harper R, Jones NM, O'Cathain A, Thomas KJ, Usherwood T, et al. Validating the SF-36 health survey questionnaire: new outcome measure for primary care. BMJ 1992;305:160-4.

17. Atherton K, Fuller E, Shepherd P, Strachan DP, Power C. Loss and representativeness in a biomedical survey at age 45 years: 1958 British birth cohort. J Epidemiol Community Health 2008;62:216-23.

18. Goldberg DP. The detection of psychiatric illness by questionnaire: a technique for the identification and assessment of non-psychotic psychiatric illness. London: Oxford University Press; 1972.

19. Chalder T. Development of a fatigue scale. J Psychosom Res 1993;37:147-53.

20. Hays RD, Bjorner JB, Revicki DA, Spritzer KL, Cella D. Development of physical and mental health summary scores from the patient-reported outcomes measurement information system (PROMIS) global items. Qual Life Res 2009;18: 873-80.

21. Kroenke K, Spitzer RL, Williams JB. The Phq-9. J Gen Intern Med 2001;16:606-13.

22. Ohayon M. Epidemiological study on insomnia in the general population. Sleep 1996;19:S7-15.

23. Wolfe F. Pain extent and diagnosis: development and validation of the regional pain scale in 12,799 patients with rheumatic disease. J Rheumatol 2003;30:369-78.

24. Pope DP, Croft PR, Pritchard CM, Silman AJ. Prevalence of shoulder pain in the community: the influence of case definition. Ann Rheum Dis 1997;56:308-12.

25. Butler S, Landmark T, Glette M, Borchgrevink P, Woodhouse A. Chronic widespread pain: the need for a standard definition. Pain 2016;157:541-3.

26. Beasley M, Macfarlane GJ. Chronic widespread pain versus multi-site pain: does the distribution matter? [abstract]. Arthritis Rheumatol 2014;66:S908-9.

27. Coggon D, Ntani G, Palmer KT, Felli VE, Harari R, Barrero $\mathrm{LH}$, et al. Patterns of multisite pain and associations with risk factors. Pain 2013;154:1769-77.

28. Yunus MB. The role of gender in fibromyalgia syndrome. Curr Rheumatol Rep 2001;3:128-34.

29. Häuser W, Kühn-Becker $H$, von Wilmoswky $H$, Settan M, Brähler E, Petzke F. Demographic and clinical features of patients with fibromyalgia syndrome of different settings: a gender comparison. Gend Med 2018;2:116-25.

30. Hooten WM, Townsend CO, Decker PA. Gender differences among patients with fibromyalgia undergoing multidisciplinary pain rehabilitation. Pain Med 2007;8:624-32.

31. Lourenço S, Costa L, Rodrigues AM, Carnide F, Lucas R. Gender and psychosocial context as determinants of fibromyalgia symptoms (fibromyalgia research criteria) in young adults from the general population. Rheumatology (Oxford) 2015;54:1806-15. 